\title{
On Ludvig Lorenz and his 1890 treatise on light scattering by spheres
}

\author{
Frisvad, Jeppe Revall; Kragh, Helge
}

Published in:

European Physical Journal H

Link to article, DOI:

10.1140/epjh/e2019-100022-y

Publication date:

2019

Document Version

Publisher's PDF, also known as Version of record

Link back to DTU Orbit

Citation (APA):

Frisvad, J. R., \& Kragh, H. (2019). On Ludvig Lorenz and his 1890 treatise on light scattering by spheres. European Physical Journal H. https://doi.org/10.1140/epjh/e2019-100022-y

\section{General rights}

Copyright and moral rights for the publications made accessible in the public portal are retained by the authors and/or other copyright owners and it is a condition of accessing publications that users recognise and abide by the legal requirements associated with these rights.

- Users may download and print one copy of any publication from the public portal for the purpose of private study or research.

- You may not further distribute the material or use it for any profit-making activity or commercial gain

- You may freely distribute the URL identifying the publication in the public portal

If you believe that this document breaches copyright please contact us providing details, and we will remove access to the work immediately and investigate your claim. 


\title{
On Ludvig Lorenz and his 1890 treatise on light scattering by spheres
}

\author{
Jeppe Revall Frisvad ${ }^{1}$ and Helge Kragh ${ }^{2, a}$ \\ ${ }^{1}$ Department of Applied Mathematics and Computer Science, Technical University \\ of Denmark, Richard Petersens Plads, Building 321, 2800 Kongens Lyngby, Denmark \\ ${ }^{2}$ Niels Bohr Institute, University of Copenhagen, Blegdamsvej 17, 2100 Copenhagen, \\ Denmark
}

Received 1 May 2019 / Received in final form 13 June 2019

Published online 30 July 2019

(C) EDP Sciences, Springer-Verlag GmbH Germany, part of Springer Nature, 2019

\begin{abstract}
This paper offers background and perspective on a littleknown memoir by Ludvig Lorenz on light scattering by spheres, which was published in Danish in 1890. It is a companion to an English translation of the memoir appearing separately. Apart from introducing Lorenz and some of his contributions to optics and electrodynamics, the paper focuses on the emergence, content and reception of the 1890 memoir and its role in what is often called the Lorenz-Mie theory. In addition to the historical analysis, the paper illuminates aspects of modern Lorenz-Mie theory and its many applications, with an eye to Lorenz's original work.
\end{abstract}

\section{Introduction}

According to Google Scholar, the term "Lorenz-Mie" appears in about 7300 scientific articles of which 5900 date from the last two decades. While the second name refers to Gustav Mie, a well-known German physicist who published his theory in 1908, the first name is much less known. In a nutshell, in a lengthy memoir of 1890 the Danish physicist Ludvig Lorenz published an elaborate optical theory on the scattering of light by small transparent spheres, which was essentially a nonelectromagnetic version of Mie's later theory. Mie's seminal paper was written in German and originally it attracted very little attention. Reflecting the growing interest in Mie's theory, the paper has been translated into English and thus made accessible to physicists and historians of physics all over the world [Wriedt 2012, p. 55; https://scattport.org/index.php/classic-papers]. Lorenz published his memoir in Danish, which effectively has made it unknown to the physics community. Only now, almost 130 years later, we can present a complete English translation of this great and complex paper which has been called "one of the most remarkable memoirs to be published in the 19th century" [Logan 1965, p. 77].

In the present paper, a companion to the translation, we discuss the historical context of Lorenz's scattering theory and relate it to some of his earlier contributions

a e-mail: helge.kragh@nbi.ku.dk 
to optics and electrodynamics. We also include a brief biographical section and outline the main trajectory that led to Mie's celebrated theory of 1908. From a historical point of view the reception of a scientific work is no less important than the work itself, and for this reason we refer to how Lorenz's theory was received by contemporary and later physicists. The second part of our paper is less historically oriented as it takes up in a more critical way some of the mathematical and physical problems appearing in or related to Lorenz's memoir. Moreover, in this part we also discuss, if only briefly and selectively, the revival of interest in Lorenz-Mie theory and aspects of its current significance and applications.

\section{Biographical sketch}

Ludvig Valentin Lorenz (1829-1891) was born in Elsinore, the city housing the castle of Shakespeare's Hamlet. "Very early on I showed an interest in calculations and mathematics," he recalled in an autobiographical note of 1877 [Kragh 2018b, pp. 243-245]. However, rather than studying mathematics or physics, as a young man he entered the Polytechnic College in Copenhagen to study applied or engineering chemistry. During his studies he followed a course in chemical physics given by the aging Hans Christian Ørsted, whose romantic natural philosophy influenced him to some degree. On the other hand, he generally preferred - contrary to Ørsted - a mathematical rather than a philosophical approach to physics.

Lorenz graduated in 1852 without distinction and without being seriously interested in his chosen field of study. Indeed, none of his later scientific works related to problems of a chemical nature. During most of the period 1852-1866 Lorenz lived a modest life as part-time teacher at middle and gymnasium schools in Copenhagen, where he taught elementary physics, chemistry and mathematics. At the same time he continued his private studies of still more advanced topics in theoretical physics. It was a turning point in Lorenz's life when he, in 1858, received grants that enabled him to travel to Paris and study physics at Sorbonne University. Attending lecture courses by illustrious physicists such as Joseph Liouville, Gabriel Lamé and Henri Regnault he was for the first time confronted with modern and advanced mathematical physics. As a direct result of his one-year stay in Paris, in 1860 he published his first scientific paper on theoretical optics. Two years later he married Agatha Fogtmann, who was to survive her husband by 31 years. The marriage was childless.

Lorenz's fortunes changed to the better only in 1866, when he obtained for the first time a permanent position as physics teacher at the Royal Military High School established in 1829. In this position, he had at his disposal an excellent laboratory and instrument collection which allowed him to engage in experimental research and not only theoretical physics. For his teaching at the Military High School, he wrote three textbooks on, respectively, general physics, optics, and heat. The book on optics was translated into German and published by the recognized Leipzig publisher B.G. Teubner [Lorenz 1877]. In the same year that Lorenz was appointed physics teacher at the Military High School, he was elected a member, at the relatively young age of 37, of the prestigious Royal Danish Academy of Sciences and Letters founded in 1742. Although Lorenz never became a professor and never wrote a doctoral dissertation, he came to be recognized as an important member of the small Danish physics community and the country's first and foremost theoretical physicist (Fig. 1). In a testimony of 1873 to the Ministry of Church and Education, the German-born professor of astronomy Heinrich Louis d'Arrest wrote as follows: "I do not hesitate to point out to the high Ministry that associate professor Lorenz is one of the first and internationally respected scientists within this field [mathematical physics]. His extremely penetrating investigations belong to the most difficult and remarkable accomplishments of our time" [Kragh 2018b, p. 238]. 


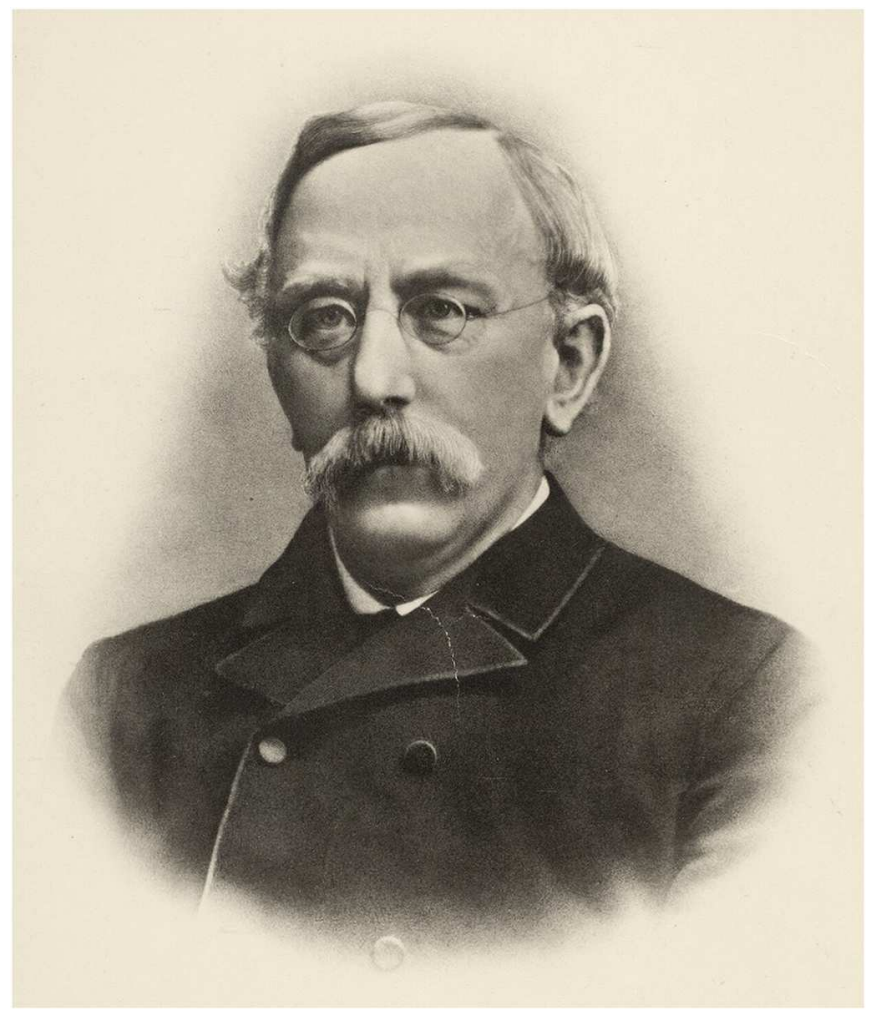

Fig. 1. Ludvig V. Lorenz. Royal Library, Copenhagen, Picture Collection.

Compared to other Danish physicists in the period Lorenz was internationally oriented and for several decades the only one with a solid reputation abroad. He was probably better known and more appreciated by physicists in German-speaking Europe than in his own country. Not only did he publish in leading physics journals such as Journal de Physique, Crelles Journal and Annalen der Physik und Chemie, he also corresponded with several of Europe's foremost physicists. Among his correspondents were distinguished scientists including Ludwig Boltzmann, Carl Neumann, Friedrich Kohlrausch, Heinrich F. Weber, August Kundt and, in France, the later Nobel Prize laureate Gabriel Lippmann. He sent regularly off-prints of his papers to these and other physicists abroad, including notables such as Anders Ångström, Marcel Brillouin, Henri Poincaré, Janne Rydberg and Hermann von Helmholtz. On the other hand, he had practically no contact to British physicists, most conspicuously not to James Clerk Maxwell and Lord Rayleigh.

While quite satisfied with his position at the Military High School, in early 1887 Lorenz received a generous offer that he could not decline. The Carlsberg Foundation established by the wealthy brewer Jacob C. Jacobsen in 1876 offered him a life-long salary as an independent scientist who could cultivate whatever field of study he found suitable. Lorenz happily accepted the offer, which meant that he had to resign his position at the Military High School and therefore also quit experimental research. During the brief span of years in which he worked as a "free scientist" he completed his ambitious analysis of the scattering of plane waves by spherical bodies about which more below. After this tour de force he focused on a work in pure mathematics dealing with prime numbers, which was to be his last publication. 
Lorenz contemplated a future work on the famous three-body problem which at the time attracted much mathematical attention. However, he did not come very far in his study of the intricate problem. On 9 June 1891 he died unexpectedly of a heart attack, at the age of 62 . While the memory of Lorenz soon faded, internationally as well as in his native country, a few years after his death the Carlsberg Foundation decided to honour him with a French edition of his collected papers. The result was Oeuvres Scientifiques de L. Lorenz edited by the mathematician Herman Valentiner and published in two volumes around the turn of the century [Lorenz 1904]. The first of the volumes, today readily available online on https://archive.org/details/ oeuvresscientifi01lore, includes the 1890 memoir.

\section{An electrical theory of light}

Whereas Lorenz's early optical theories were within the established tradition of the elastic theory of light, in 1867 he proposed a new theory in which light waves were considered to be oscillating electrical currents and not vibrations in an ethereal medium. As he wrote, there was "scarcely any reason for adhering to the hypothesis of an ether, for it may well be assumed that in the so-called vacuum there is sufficient matter to form an adequate substratum for the motion" [Lorenz 1867]. The theory was in part inspired by the dynamical ideas of Ørsted, his former teacher, which he developed into a concise mathematical theory. Building on an earlier theory of the propagation of electricity in conducting media proposed by Gustav Robert Kirchhoff (1857), but unaware of Maxwell's recent electromagnetic theory of light, Lorenz arrived at equations for the variation of the current density $\mathbf{j}$. In a condensed form and with $\sigma$ denoting the electrical conductivity, his wave equation for $\mathbf{j}$ can be expressed as

$$
-\nabla \times(\nabla \times \mathbf{j})=\frac{1}{a^{2}} \frac{\partial^{2} \mathbf{j}}{\partial t^{2}}+\frac{16 \pi}{a^{2}} \sigma \frac{\partial \mathbf{j}}{\partial t}
$$

where $a$ is the velocity of the electrical wave (or light wave). Lorenz identified the last term with the absorption of light which would increase with the conductivity. It is to be noted that there were no electrical or magnetic fields in the 1867 theory and nor were there any displacement currents. All electrical currents and hence also light were conduction currents.

Although Lorenz's electrodynamic theory of light has long ago been abandoned, two of the important results of the 1867 paper are still parts of modern physics. Lorenz emphasized that the action of electrical disturbances requires time to propagate corresponding to a finite velocity as given by retarded potentials. Although the general idea of retarded electrical action had previously been suggested (by C. Friedrich Gauss and Bernhard Riemann, see Kaiser (1981)), Lorenz was the first to incorporate the idea into a coherent theory of light and electricity. For a point $\mathbf{x}=(x, y, z)$ in a volume $V$, his formula for the scalar potential due to a charge density $\rho$ was

$$
\varphi(\mathbf{x}, t)=\int \frac{\rho\left(\mathbf{x}^{\prime}, t-\frac{r}{a}\right)}{r} d V
$$

where $r=\left|\mathbf{x}-\mathbf{x}^{\prime}\right|$ and $d V=d x^{\prime} d y^{\prime} d z^{\prime}$. Similarly, Lorenz's formula for the vector potential A was

$$
\mathbf{A}(\mathbf{x}, t)=\int \frac{\mathbf{j}\left(\mathbf{x}^{\prime}, t-\frac{r}{a}\right)}{r} d V
$$


The other technical innovation appearing in Lorenz's paper concerned the relationship between the retarded potentials. Following Kirchhoff's notation, he originally used $\bar{\Omega}$ for the scalar potential and $(\alpha, \beta, \gamma)$ for the vector potential, arguing that they had to satisfy the constraint

$$
\frac{\partial \bar{\Omega}}{\partial t}=-2\left(\frac{\partial \alpha}{\partial x}+\frac{\partial \beta}{\partial x}+\frac{\partial \gamma}{\partial x}\right) .
$$

In modern notation and Gaussian units, the factor 2 disappears and the constraint becomes

$$
\nabla \cdot \mathbf{A}+\frac{1}{c} \frac{\partial \varphi}{\partial t}=0
$$

This is the famous Lorenz condition, by far the most useful of the various gauges used in electrodynamics. Until recently the gauge stated in 1867 was predominantly named the "Lorentz gauge" because Hendrik A. Lorentz used it in his important work on electromagnetic theory in the early years of the twentieth century. Contrary to the Coulomb gauge $\nabla \cdot \mathbf{A}=0$, this gauge is manifestly Lorentz invariant. Although "Lorentz gauge" is still widely used, paternity belongs to Lorenz of Copenhagen [Jackson and Okun 2001].

Lorenz's electrical theory of light was well known to contemporary physicists and referred to by, for example, Maxwell, Heinrich Hertz, Éleuthère Mascart, Carl Friedrich Zöllner, and Oliver Lodge. In the early part of the twentieth century it was critically discussed by Pierre Duhem. However, by that time it had largely fallen into oblivion. We shall not here examine the fate of Lorenz's electrical oscillation theory of light, for which we refer to the recent literature [Keller 2002; Kragh 2018b,c]. An important reason for the cool reception and later neglect was undoubtedly that Lorenz did not follow up upon it or refer to it in his later works. Nor did he ever refer to Maxwell's field theory of light and its relationship to his own. It is remarkable that there is no trace of electromagnetism in Lorenz's great work on the scattering of light by spheres. The memoir of 1890 contains no reference to his earlier theory and words such as "electrical" and "magnetic" do nowhere appear significantly.

\section{Optical theories, ca. $1860-1883$}

Influenced by his research stay in Paris, Lorenz's first scientific papers were based on the elastic or mechanical theory of light which still in the early 1860s constituted the generally accepted theoretical framework in optics. According to this framework or paradigm, light consisted of transverse waves made up of deformations in an incompressible ethereal medium [Whittaker 1958, pp. 128-170; Darrigol 2012, pp. 225-239]. About 1860, when Lorenz entered the field, the incompressibility of the solid ether and the transverse nature of the vibrations therein were subjects of debate which Lorenz's early works in optics helped to clarify. Most of these works appeared in Annalen and were reviewed in Fortschritte der Physik, the abstract journal published since 1845 by the German Physical Society. They were thus well known to the international community of physicists.

With the purpose of understanding the ether vibrations in the reflection and refraction of light, Lorenz (1861) assumed that the two optical media were separated by a boundary or transition layer. This layer he imagined to be divided in an infinite number of infinitesimally thin sheets, each of the sheets having a constant density. He estimated that the thickness of a typical layer was between 1 and 10 per cent of 
the wavelength of visible light or roughly between $5 \mathrm{~nm}$ and $50 \mathrm{~nm}$. Lorenz's concept of an optical transition layer was original and later recognized to be an important innovation, but at the time it was either criticized or ignored [Schuster 1909, p. 241; see also Keller 2002]. The notion of a continuum of transition layers reappeared in Lorenz's later scattering theory, now adapted to spherical surfaces. With $\omega$ denoting the velocity of the light, the hypothesis entered as follows: "This discontinuous transition is considered to be produced by a surface layer of finite thickness and continuous change of $\omega$... that approaches a layer of thickness zero" [Lorenz 1890, pp. 4-5].

After less than two years of work within the mechanical-elastic paradigm, Lorenz realized that the laws of optics could not be fully deduced from the theory of elasticity. Adopting an alternative non-mechanical methodology, he now concluded that the fundamental theory of light must rest solely on abstract conceptions in agreement with observed phenomena. The "phenomenological" approach implied that the physical nature of light was disregarded and also that the ether was given less attention. Although the ether still figured in Lorenz's papers until 1865, it was merely as a name without physical characteristics. In a paper of 1863, he introduced an abstract light vector $\mathbf{u}=\left(u_{x}, u_{y}, u_{z}\right)$ on the basis of which he formulated three differential equations [Lorenz 1863]. He claimed that in heterogeneous, non-absorbing media all phenomena of light can be deduced from these equations, which in modern notation can be written as

$$
-\nabla \times(\nabla \times \mathbf{u})=\frac{1}{a^{2}} \frac{\partial^{2} \mathbf{u}}{\partial t^{2}}
$$

Lorenz had $a=\omega / k$, where $\omega$ is the angular frequency and $k$ is the wave number. This is equivalent to $a=c / n$, where $c$ is the speed of light in vacuo and $n$ is the index of refraction. The same differential equations entered into Lorenz's 1867 electrical theory of light, except for the added absorption term in the latter theory. The equations also formed the basis of his 1890 scattering theory. "The differential equations from which the present investigation takes its starting point," Lorenz stated in his memoir (p. 3), "differ from the theory of elasticity by the fact that they rule out the possibility of longitudinal oscillations, and, since they are valid for every point in any transparent heterogeneous medium, the boundary conditions at the transition from one body to another can be derived from the differential equations themselves." It should be mentioned that much earlier the Irish physicist James MacCullagh had derived a wave equation similar to Lorenz's, but MacCullagh's theory rested on the assumption of an elastic solid ether [Darrigol 2010].

In a paper of 1869, Lorenz derived on the basis of experiments and his optical wave theory a relationship between a transparent body's refractivity index $N$ and its specific volume $v$ as given by $v=1 / d$, where $d$ is the density. He concluded that

$$
\frac{N^{2}-1}{N^{2}+2} v=\text { constant. }
$$

For reasons of simplicity, he assumed the refractive medium to be composed of optically homogeneous spherical molecules with $N_{i}$ being their internal refractive index. With $v_{i}$ being the specific proper volume of the molecules, Lorenz could then state the law as

$$
\frac{N^{2}-1}{N^{2}+2} v=\frac{N_{i}^{2}-1}{N_{i}^{2}+2} v_{i} .
$$

Nine years later, 25-year-old H. A. Lorentz, who had recently been appointed professor at the University of Leiden, derived the same refractivity formula but on the basis 
of the electromagnetic theories of Maxwell and Helmholtz. Since Lorentz's paper was in Dutch, and Lorenz's in Danish, the "Lorentz-Lorenz law" became widely known only after 1880, when revised versions of the two memoirs appeared in German translations [Lorentz 1880; Lorenz 1880]. As to Lorenz, he used his refraction-density theory to estimate a lower limit to the size of molecules. For what he called "the radius of the molecular sphere of action," a quantity greater than the actual radius of the molecule, he found its lower limit to be $15 \mathrm{~nm}$. Lorenz returned to the question of the size of molecules in the last sections of his 1890 memoir. More information about the early history of what is known as either the Lorentz-Lorenz or the Lorenz-Lorentz law can be found in Kragh (2018a).

Finally, among the sources for Lorenz's scattering theory of 1890 was also an extensive paper on chromatic dispersion published in Annalen [Lorenz 1883; Keller 2002, pp. 283-285]. As Lorenz (1890, p. 3) pointed out in his introduction, this paper served as the direct inspiration for his more elaborate theory of the scattering of plane light waves by a transparent and isotropic sphere. Moreover, it contained mathematical concepts and methods that would appear in his memoir seven years later. Although the basic equations of the 1890 treatise were the same as those employed in his dispersion theory of 1883, Lorenz (1890, p. 3) now "preferred a different and simpler way of presentation where I, also to ease the reading, shall avoid assuming knowledge of my previous work."

The 1883 dispersion theory assumed a collection of randomly ordered point atoms surrounded by concentric shells. Each shell was characterised by a constant refractive index, which diminished with its distance from the atom, corresponding to a variation of the effective velocity of light. Based on this model Lorenz derived a dispersion relation, but unfortunately it was unable to account for anomalous dispersion. For this and other reasons his dispersion relation was considered to be unsatisfactory.

\section{From Clebsch to Mie}

Lorenz presented his new scattering theory to the Royal Danish Academy in an address of 18 October 1889 in which he called attention to an earlier work by the German mathematician and physicist Adolf Clebsch:

I need to mention a single attempt to determine the reflection of light from a perfectly bright spherical surface ... been made by Clebsch but with the deterrent results, as he puts it in the explanations to his great work (Crelles Journal of 1863), that the results of the entire investigation are terribly complex.... On the other hand, he has succeeded in the case of very small reflecting spheres. The mentioned author proceeds from the differential equations of elasticity theory, [while] ... I have myself based the theory on different equations, the same which I proposed for approximately 25 years ago. [Kragh 2018b, p. 104]

Clebsch died in 1872, only 46 years old. As assistant professor at Karlsruhe Polytechnic College he played an active role in the German mathematical community, contributing with innovative ideas in both pure mathematics and theoretical physics. For example, in 1866 he wrote a mathematical treatise on Abelian functions jointly with Paul Gordan. The Clebsch-Gordan coefficients used extensively in quantum mechanics and elementary particle physics have their roots in this treatise. More to the point, what caught Lorenz's attention was a mathematically challenging 68 page memoir on the reflection of elastic waves by a spherical obstacle [Clebsch 1863; Todhunter 1893, Part 2, pp. 168-180; Logan 1965]. Although published in one of 
the period's most important mathematical journals, Crelles Journal, Clebsch's preMaxwell memoir exerted almost no interest at all on either the mathematicians or the physicists. In fact, during the late nineteenth century Lorenz was alone in recognizing its importance and receiving inspiration from it. However, since Lorenz's Danish treatise remained practically unknown, its highlighting of Clebsch's work made no difference.

Apart from Clebsch's 1863 memoir, Lorenz also cited earlier works by George Stokes, George Airy, Lord Rayleigh (or John William Strutt) and a few other authors. Of Rayleigh's extensive writings on the scattering of waves he only cited an early set of papers based on the equations of elastic waves in which Rayleigh (1871a,b,c) had derived the scattering law for small particles. Although Lorenz was presumably aware of Rayleigh's work of 1881 [Kerker 1969], which offered an electromagnetic derivation of the scattering law, he did not refer to this work and also not to other works based on Maxwell's theory of light. At the end of his memoir, Lorenz considered the case of small spheres of molecular dimensions. He proved that the scattering cross section of a single sphere of radius $R$ very small compared to the wavelength $\lambda$ is

$$
C_{s}=\frac{128}{3} \frac{\pi^{5} R^{6}}{\lambda^{4}}\left(\frac{N^{2}-1}{N^{2}+2}\right)^{2} .
$$

This equation provides both the scattering cross section of a small particle and its link to the polarizability of a spherical particle as given by the Lorentz-Lorenz relation. Since the refractive index for air and other transparent bodies only varies slightly in the wavelength range of visible light, this expression for $C_{s}$ is dominated by the $\lambda^{-4}$ term. As Lorenz pointed out, this result agreed with what Rayleigh had found in 1871 . For $N$ close to 1 , it gives approximately the same expression as found by Rayleigh, namely

$$
C_{s}=\frac{128}{27} \frac{\pi^{5} R^{6}}{\lambda^{4}}\left(N^{2}-1\right)^{2}
$$

In his later theory of scattering, Rayleigh (1899) explains that his original scattering cross section is only valid for $N$ close to 1 as the shape of the particle must be taken into account in the preferred electric theory [Rayleigh 1881]. He continues to say that $N^{2}-1$ should be replaced by $3\left(N^{2}-1\right) /\left(N^{2}+2\right)$ in the case of spheres. The two theories then agree very well. The remaining difference between them is Rayleigh's (1871a) simple expression for the distribution of the scattered light (the Rayleigh phase function), where Lorenz has the fully general solution for a sphere. Rayleigh's theory is in other words for particles significantly smaller than the wavelength with spheres as a special case, whereas Lorenz's theory is for spheres of arbitrary size with small spheres as a special case.

Rayleigh (1899) also derived an expression for the number density of scattering particles $N_{p}$ in terms of the refractive index $N$ of the gas in question:

$$
N_{p}=\frac{32 \pi^{3}}{3 h \lambda^{4}}(N-1)^{2} .
$$

Here, $h$ is the extinction coefficient (scattering plus absorption), which is an observable quantity. For non-absorbing particles, the extinction coefficient is only a measure of the amount of scattering per unit of distance as light travels through the medium, and then $h=C_{s} N_{p}$. Lorenz referred to $h$ as the absorption coefficient. For an ideal gas under standard conditions, the molecules become the scatterers and $N_{p}$ corresponds to Loschmidt's number $N_{L}$. Loschmidt's number relates to Avogadro's number 
through

$$
N_{A}=6.022 \times 10^{23} \mathrm{~mol}^{-1} \approx N_{L} \times 22.4 \times 10^{3} \mathrm{~cm}^{3} \mathrm{~mol}^{-1} .
$$

Lorenz's formula as given in the 1890 memoir was

$$
N_{p}=\frac{24 \pi^{3}}{h \lambda^{4}}\left(\frac{N^{2}-1}{N^{2}+2}\right)^{2} .
$$

If $N$ is only slightly larger than one, which is certainly the case for a gas, Lorenz's expression is fairly similar to the one stated by Rayleigh nine years later. Lorenz illustrated his theory by considering a concrete example where plane waves of light of wavelength $\lambda=580 \mathrm{~nm}$ pass through atmospheric air. In this case, he found the value $N_{p}=1.63 \times 10^{19} \mathrm{~cm}^{-3}$, which is more than half of $N_{L}$. For the case of spherical particles, several of the results communicated by Rayleigh in 1899 can be found in Lorenz's earlier work. Considering the historical development of the theory of scattering by a sphere, Milton Kerker (1969, p. 59) concludes that "certainly if this theory is to be associated with the name or names of individuals, at least that of Lorenz, in whose paper are to be found the practical formulas so commonly used today, should not be omitted." Rayleigh only referred to Lorenz's theory in 1918, in the autumn of his life, and then only briefly.

Contrary to Lorenz, when Gustav Mie in 1908 published his now very famous treatise on the scattering by conducting spheres, he did not refer to Clebsch's memoir. Nor did he cite Lorenz's paper in either its Danish original or the French translation available since 1898. Mie's theory has recently been the subject of historical attention and in the present context there is no need to go into details, as these can be found in the literature [Horvath 2009; Hergert 2012; Wriedt 2012]. At the time professor at the University of Greifswald, Mie was an expert in Maxwellian electrodynamics and a leading figure in the ill-fated, pre-Einsteinian attempts to establish this theory as the foundation of all of physics [Vizgin 1994]. Today he may best be known for his 1908 scattering theory, which more specifically was a theory of colour effects of colloidal metal solutions, but he did not himself consider it to be particularly important. Nor did most other physicists, for originally it only attracted modest attention such as indicated by the very few references to it until about 1970 (Fig. 2). What matters here is the relation of Mie's paper to Lorenz's, which today is widely seen as an anticipation of it or even an equivalent but physically different version of Mie's electromagnetic theory.

Although Mie did not formally cite Lorenz's paper on scattering by a sphere, he cited Lorenz (1880) on the refractivity-density law and may even have known of his 1890 memoir if without studying it in any detail [Kragh 2018b, pp. 115-116]. Whatever the historical connection between the two papers, today it is widely recognized that they are empirically equivalent. As part of his Munich doctoral dissertation Peter Debye (1909a) wrote an important paper on the scattering problem, including an analysis of the light pressure exerted on a sphere, in which he cited the earlier papers of Clebsch and Mie. Although he did not refer to Lorenz's memoir, he was aware of it and had studied it in its French translation. This is evidenced by another paper of a predominantly mathematical nature in which Debye (1909b) referred to formulae first stated by Lorenz.

While Lorenz's theory was abstract and formulated without a physical interpretation of light and its propagation through space, Mie's was solidly founded on the Maxwell-Lorentz theory of electromagnetism. According to Gouesbet (2012, p. 74), Lorenz's scattering theory rested on the assumption of the ether, while Mie's did not. Horvath (2009) likewise states that "Lorenz completely solved for the scattering 


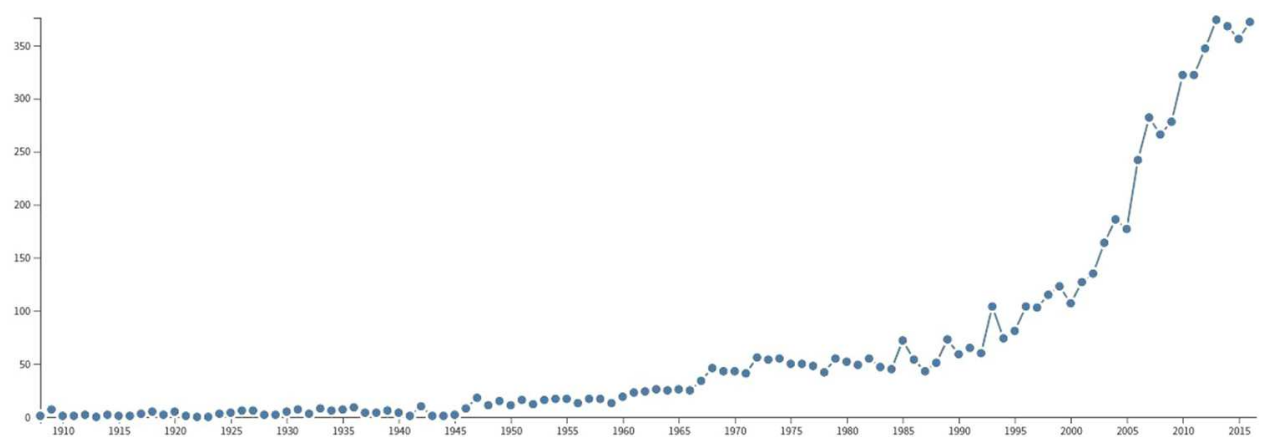

Fig. 2. Number of citations to G. Mie's 1908 paper as a function of time according to Web of Science.

of light by small particles theoretically using the ether theory." However, this is a misunderstanding. It was Mie's electromagnetic theory, not Lorenz's, which relied on the ether, and it did so because it was based on the Maxwell-Lorentz ether theory. The ether played no role at all in Lorenz's memoir, where the word "ether" simply did not appear.

Lorenz made his theory of light "free from all physical hypotheses" to prevent that the mathematical model would be nullified by "further progress of a future time" [Lorenz 1867]. This turned out to be a good decision as his solution for the scattering of light by a sphere from 1890 is mathematically equivalent to those of Mie and Debye. The reason is that, for a non-magnetic substance (permeability equal to unity), the exact same wave equation as the one used by Lorenz (1) can be derived for the electric vector using Maxwell's equations [Born and Wolf 1999, p. 11]. Moreover, for a plane wave and a spherical wave, the magnetic vector is not required. We only need the electric vector and the direction of wave propagation to determine the Poynting vector and thus the distribution of radiant energy. In other words, Lorenz selected the exactly right phenomenological model for his theoretical investigations, and we can therefore directly use his results even today.

The term Lorenz-Mie theory as a substitute for Mie theory first turned up in the early 1960s [Wait 1962; Logan 1962]. As stated by Wait (1962), "The theory of scattering of electromagnetic waves from a homogeneous sphere was first given in explicit form by Lorenz and some time later by Mie." Strictly speaking this is of course incorrect, since Lorenz's theory was not about electromagnetic waves. Despite the increased recognition of Lorenz's contribution, it is still more common to speak of the Mie theory, whereas Mie-Lorenz theory is rarely used. According to Google Scholar a total of ca. 92,300 papers refer to Mie theory and ca. 4930 to Lorenz-Mie theory. For the period 1980-2018 the corresponding numbers are ca. 42,700 and ca. 4780. Out of these 4780 scientific articles, around one third are referring to generalized Lorenz-Mie theory (GLMT, more on this theory later). GLMT has thus been quite influential with respect to inclusion of Lorenz's name.

\section{Contents of Lorenz's memoir in brief}

Lorenz started his 1890 memoir by referring to Clebsch (1863) and to his own previous work [Lorenz 1883]. He also set the scene by considering the differential equations (1) of his previous work the model of light propagation (the variable we denote by $a$ is denoted by $\omega$ in Lorenz's papers). All his results were derived from this model of light propagation. Throughout the memoir, Lorenz specifically considered the interaction 
of a plane wave with a spherical surface. Like Mie (1908) and Debye (1909a) after him, he solved the differential equations describing the light propagation by reformulating the problem in spherical coordinates. The incident plane wave was expanded in spherical harmonics (using a formula first found by Bauer (1826)), and the boundary conditions were used to obtain the spherical harmonics expansion coefficients of the scattered wave. Numerical evaluation of the solution was difficult, so Lorenz spent many pages developing a theory for approximate evaluation of special cases.

The size parameters for a particle of radius $R$ and relative index of refraction $N$ are

$$
x=\frac{2 \pi R}{\lambda} \text { and } y=\frac{2 \pi R N}{\lambda}
$$

where $\lambda$ is the wavelength in vacuo. Lorenz used $\alpha$ and $\alpha^{\prime}$ to denote $x$ and $y$ and considered the special cases of $\alpha$ very large and $\alpha$ very small, that is, large and small particles as compared with the wavelength. He also included a section on the special case of light getting trapped in a thin layer inside the sphere due to total internal reflection (when $N<1$ ). Lorenz used the case of $\alpha$ very large to discuss the appearance of rainbows and the case of $\alpha$ very small to discuss absorption lines in systems of particle and similarities of his theory with that of Rayleigh.

Lorenz considered the right mathematical model (as explained in the previous section) and was therefore able to derive accurate formulae for the amount and the intensity of the scattered light. However, since he did not offer a physical hypothesis, he could not draw electric and magnetic field lines of partial waves like Mie did. Mie (1908) solved the differential equations separately for the electric and magnetic vectors. Then, based on Poynting's theorem [Poynting 1884], he found that the solution for the electric vector was sufficient "to obtain a clear representation of the radiation." Based on Mie's observations, Debye (1909a) showed that we only need to solve differential equations for a pair of scalar potentials. From these, we can calculate the full solution for the electric and magnetic vectors outside the sphere. Although Lorenz considered the wave vector corresponding to the electric vector only, he found the same pair of scalar potentials. In addition, he had a pair of potentials relating to the scattered wave inside the sphere.

Interestingly, Mie (1908) mentioned that his theory could be used for the rainbow problem. However, he found that this would require taking into account a rather large number of partial waves, which would result in "considerable difficulties in calculation." This explains Lorenz's need for lengthy calculations to approximate the case of $\alpha$ very large.

\section{Context and reception of the 1890 theory}

Lorenz's great work was published in Danish in the not widely circulated transactions of the Royal Danish academy and for this reason alone it remained unknown or at least inaccessible to the international community of physicists. At the turn of the century it was available in French translation, but Oeuvres Scientifiques de L. Lorenz was rarely read and failed to make the theory more generally known. Although the language barrier was undoubtedly a major reason for its lack of visibility, it was no less important that the theory ignored what at the time was considered modern field electrodynamics. By the late 1890s Maxwell's ether-based theory of electromagnetism completely dominated physicists' thinking about optics, so why pay attention to a complex and obscure theory of the past that failed to take into account electromagnetic fields and the associated ether? Kerker (1969, p. 56) ascribes the neglect of Lorenz's theory to be in part that "His solution is based upon his own theory of electromagnetism rather than on that of Maxwell." The word "electromagnetism" 
should probably have been "light propagation" in this sentence since it was Lorenz's 1863 theory of light propagation rather than his 1867 electrical theory of light that played a role in his later scattering theory.

In spite of the language barrier and the non-electromagnetic framework of Lorenz's scattering theory, it was not entirely neglected. For the few early references to the theory, see Logan (1965) and Kragh (2018b, pp. 112-115). It is worth noting that the British physicist J.C. Maxwell Garnett (1904) in an important paper on the colour of metals cited Lorenz's Danish memoir and that Mie four years later cited Maxwell Garnett. By the way, Maxwell Garnett (1880-1958) was not related to the famous J.C. Maxwell although he was named after him. Also worth noting is that H.A. Lorentz never mentioned Lorenz's 1890 theory or worked on the optical scattering problem. Nonetheless, in a textbook of atmospheric optics we read that Mie's theory "was anticipated by Lorentz during the period 1890 to 1900" [McCartney 1976, p. 217]. The author possibly had Lorenz in mind but may have confused him with the famous Dutch physicist, such as was commonly done (and is still done) in connection with other of Lorenz's contributions and with the Loren $(\mathrm{t}) \mathrm{z}$ gauge condition in particular. It is more than a little disturbing to find in the physics literature about 740 references to the non-existing "Lorentz-Mie theory."

Throughout his life Lorenz nourished a burning interest in mathematics whether of the pure form or as applied to problems of physics [Kragh 2018b, pp. 207-215]. Indeed, to the limited extent that Lorenz's work of 1890 was known in the early part of the twentieth century it was more because of its mathematical rather than its physical content. When Debye in 1909 cited Lorenz's memoir it was because of the mathematical formulae it contained, and the same was the case with several other citing authors. Harry Bateman, a talented mathematician and theoretical physicist, was aware of Lorenz's memoir to which he referred in a book on electrical and optical waves. As Bateman (1915, p. 79) noted, for the case of a spherical body Lorenz had "long ago" studied the diffraction problem in relation to the rainbow. Some years earlier Bateman's compatriot, the mathematical physicist John Nicholson (1910), studied exact solutions of how waves are scattered by a sphere, and in this connection he discussed asymptotic formulae for Bessel functions with reference to results found in Lorenz's 1890 memoir. In this paper, Lorenz had derived a formula for the asymptotic expansion of products of Bessel functions of the form $J_{\alpha}^{2}+Y_{\alpha}^{2}$, where the two symbols refer to Bessel functions of the first and the second kind. The result attracted the attention of the Cambridge mathematician George N. Watson, who in his monumental treatise on Bessel functions credited Lorenz with the discovery of a formula which previously had been thought to date from 1906 [Watson 1922, p. 22 and p. 29]. Lorenz's expansion formula appears as equation (66) in his 1890 memoir and the quantities introduced by him are currently known under the name Lorenz-Mie coefficients. Logan (1965), Kerker (1969), and Wait (1998) refer to other mathematical innovations appearing in Lorenz's "lost memoir."

\section{Lorenz-Mie theory then and now}

The usefulness of an analytic solution for an idealized special case like the scattering of a plane wave by a perfect sphere is remarkable. When developing more general scattering theory, it is good practice to validate against limiting cases such as a perfect sphere or a plane wave. This is one of the key reasons why Lorenz's work of 1890 has remarkable longevity and is still being cited more than five quarter-centuries after its publication. Another key reason behind the longevity of the Lorenz-Mie theory is its ability to predict scattering effects when particles are somewhat like spheres. Prediction of rainbow effects [Lorenz 1890] and the brilliant colours of colloidal metal 
solutions [Mie 1908] are just examples. A review by Kerker (1982) illustrates the richness of the Lorenz-Mie theory in explaining scattering phenomena. At this point in time (1982), almost a century after Lorenz published his work, Kerker points out that "within the lode of the Lorenz-Mie equations [...] most of the treasures still to be mined have not yet been brought to view." Even now, the Lorenz-Mie theory seems to hold hidden treasures.

In various parts of Lorenz's 1890 treatise, his conclusive equations are surprisingly close to the equations appearing in modern articles and textbooks on scattering of electromagnetic radiation (or light). Let us consider Lorenz's exposition of the LorenzMie coefficients, which reappear again and again in generalized versions of the theory. Naming conventions for special functions were of course not quite as firmly settled in the late 19th century, so Lorenz provided mathematical definitions. For example, Lorenz defined Legendre polynomials $\left(P_{n}\right)$ using a recurrence relation (last equation of page 8) and used them for expansion just like in a modern text book [Arfken et al. see 2013, for example]. As in most later expositions of Lorenz-Mie theory, Lorenz expanded the complex exponential appearing in the wave equations and found that a variation of Bessel functions provided a solution for the scattered wave. The variation is today called Riccati-Bessel functions $\psi_{n}$ and $\zeta_{n}$, and these are typically defined in terms of spherical Bessel functions $j_{n}$ and $y_{n}$. Lorenz used $v_{n}$ and $w_{n}$, which we can connect to the Riccati-Bessel and spherical Bessel functions as follows:

$$
v_{n}(z)=\psi_{n}(z)=z j_{n}(z) \quad \text { and } \quad v_{n}(z)+w_{n}(z) \mathrm{i}=\zeta_{n}(z)=z j_{n}(z)-\mathrm{i} z y_{n}(z) .
$$

Lorenz's equations (33) for the Lorenz-Mie coefficients are

$$
\begin{aligned}
& 2 k_{n}=-1-\frac{\left(v_{n}(\alpha)-w_{n}(\alpha) \mathrm{i}\right) v_{n}^{\prime}\left(\alpha^{\prime}\right)-N\left(v_{n}^{\prime}(\alpha)-w_{n}^{\prime}(\alpha) \mathrm{i}\right) v_{n}\left(\alpha^{\prime}\right)}{\left(v_{n}(\alpha)+w_{n}(\alpha) \mathrm{i}\right) v_{n}^{\prime}\left(\alpha^{\prime}\right)-N\left(v_{n}^{\prime}(\alpha)+w_{n}^{\prime}(\alpha) \mathrm{i}\right) v_{n}\left(\alpha^{\prime}\right)}, \\
& 2 s_{n}=-1-\frac{N\left(v_{n}(\alpha)-w_{n}(\alpha) \mathrm{i}\right) v_{n}^{\prime}\left(\alpha^{\prime}\right)-\left(v_{n}^{\prime}(\alpha)-w_{n}^{\prime}(\alpha) \mathrm{i}\right) v_{n}\left(\alpha^{\prime}\right)}{N\left(v_{n}(\alpha)+w_{n}(\alpha) \mathrm{i}\right) v_{n}^{\prime}\left(\alpha^{\prime}\right)-\left(v_{n}^{\prime}(\alpha)+w_{n}^{\prime}(\alpha) \mathrm{i}\right) v_{n}\left(\alpha^{\prime}\right)} .
\end{aligned}
$$

Using the more common symbols for the Riccati-Bessel functions and through simple rearrangement of the equations, we arrive at the Lorenz-Mie coefficients as they appear in classic textbooks [van de Hulst 1957; Kerker 1969]:

$$
\begin{aligned}
& -k_{n}=\frac{\psi_{n}(\alpha) \psi_{n}^{\prime}\left(\alpha^{\prime}\right)-N \psi_{n}^{\prime}(\alpha) \psi_{n}\left(\alpha^{\prime}\right)}{\zeta_{n}(\alpha) \psi_{n}^{\prime}\left(\alpha^{\prime}\right)-N \zeta_{n}^{\prime}(\alpha) \psi_{n}\left(\alpha^{\prime}\right)}=a_{n}, \\
& -s_{n}=\frac{N \psi_{n}(\alpha) \psi_{n}^{\prime}\left(\alpha^{\prime}\right)-\psi_{n}^{\prime}(\alpha) \psi_{n}\left(\alpha^{\prime}\right)}{N \zeta_{n}(\alpha) \psi_{n}^{\prime}\left(\alpha^{\prime}\right)-\zeta_{n}^{\prime}(\alpha) \psi_{n}\left(\alpha^{\prime}\right)}=b_{n},
\end{aligned}
$$

where the prime' denotes derivative when applied to one of the spherical functions, $N$ is the relative index of refraction, and $\alpha$ and $\alpha^{\prime}$ are the size parameters (often denoted $x$ and $y$, as mentioned in Sect. 6). The only differences between Lorenz's coefficients and the modern ones $a_{n}$ and $b_{n}$ are choice of symbols and choice of sign. Lorenz probably extracted -1 from the fractions to ease approximation in a time without computers.

Debye (1909a) reproduced the theory of Mie (1908) using scalar potentials, which is now the method of choice in such derivations. These scalar potentials are today called Debye potentials, but the same scalar potentials were used by Lorenz in 1890 (as also mentioned by Kerker (1969) and in Sect. 6). Debye's purpose was different from Lorenz's and Mie's. He wanted to investigate the radiation pressure on small spheres due to the momentum generated when an incident field is scattered or absorbed. However, like Mie (1908), Debye only derived the expansion coefficients for 
the scattered wave and neglected effects due to heat distribution inside the spherical particle. His theory therefore cannot explain photophoresis, which is the motion of absorbing particles in a fluid medium [Kerker 1982]. To consider the heat distribution inside a spherical particle and its influence on photophoretic force [Kerker and Cooke 1973, 1982], the interior wave suddenly becomes of interest.

Some of the first generalizations of the Lorenz-Mie theory were to explain scattering by coated or multilayered (concentric) spheres [Aden and Kerker 1951; Kerker 1969]. In such generalizations, the expansion coefficients change while the rest of the scattering theory remains more or less unchanged. The Lorenz-Mie coefficients then serve as a limiting case where the coating and the particle have the same index of refraction. On the other hand, if we consider other heterogeneities inside the sphere, the interior wave again becomes of interest. This is the case, for example, if we would like to study cell biology and consider molecular scattering [Chew et al. 1976] or a distribution of absorption centers [Dusel et al. 1979] within an irradiated sphere. The expansion coefficients of the interior wave $\left(c_{n}\right.$ and $\left.d_{n}\right)$ were derived by Kerker and Cooke (1973) and in later work by other authors, often with an unnecessarily complicated numerator. Chew et al. (1976) found the simpler version of the numerator $(\mathrm{Ni})$, but it is worth noting that these expansion coefficient were given by Lorenz in 1890 including the simple numerator [Lorenz 1890, Eqs. (34)]. Thus the complete treatment of the interior wave together with the scattered wave distinguishes Lorenz's work from other early work on the scattering of a plane wave by a sphere.

An important generalization of the Lorenz-Mie theory is to consider an arbitrary position of the scattering sphere in an arbitrarily shaped electromagnetic beam [Gouesbet and Gréhan 2017]. The first version of this generalized Lorenz-Mie theory was developed in the 1980s by Gouesbet and colleagues [Gouesbet and Gréhan 1982; Gouesbet et al. 1988]. In this more general setting, one would use the original Lorenz-Mie coefficients multiplied by correction factors (same factors for $a_{n}$ and $c_{n}$ and for $b_{n}$ and $d_{n}$ ). Citing Dusel et al. (1979), the interior wave was introduced (without simplification) into the generalized theory by Barton et al. (1988). The intensity distribution of the interior wave is required for explaining explosion characteristics of droplets exposed to a very narrow laser beam [Schaub et al. 1989]. As another example, this internal intensity distribution is needed for reconstruction of the 3D temperature field within a combusting droplet [Castanet et al. 2005]. Gouesbet and Gréhan (2017, Sect. 8.2) provide an overview of research employing generalized Lorenz-Mie theory to calculate resonances in the internal field of a particle situated in a laser beam. They also explain (in Sect. 3.6) that it is possible to derive the simple numerator for the Lorenz-Mie coefficients of the interior wave using a Wronskian relation. A fact discovered by Lorenz in his work of 1890, but largely overlooked even today.

Another generalization of the Lorenz-Mie theory is to consider an absorbing host medium. As described by Mie (1908), Maxwell's equations reveal that a nonzero conductivity leads directly to an imaginary part in the refractive index of the material, and this makes the material an absorbing medium. Early on, a derivation of the Lorenz-Mie theory by Stratton (1941) showed that the Lorenz-Mie coefficients have the same mathematical expressions regardless of the absorption/conduction properties of the sphere and the medium. The only difference is whether the refractive indices are real or complex numbers. This means that, regardless of the absorption properties of the particle and the host, the mathematical equations for computing the Lorenz-Mie coefficients are as described by Lorenz in 1890. The output from the Lorenz-Mie theory, however, can be quite different depending on whether the refractive index of the host or the sphere is real-valued or complex-valued. The net effect of light scattering by particles thus changes depending on the absorption properties of the host medium and the particles.

Lorenz (1890) considered a transparent sphere in a transparent medium, which is very useful for explaining atmospheric light scattering phenomena like sky, rainbows, 
and clouds. In this case, the refractive index of both particle and host are real numbers. Mie (1908) investigated an absorbing sphere in a transparent host to explain the colourful scattering by colloidal metal solutions. An absorbing host is needed for explaining the appearance of media like milk, icebergs, and natural waters [Frisvad et al. 2007b]. Milk serum and water and ice are all weakly absorbing host media in these examples, like in many others. Light scattering by the atmosphere and by sea ice and seawater (and soot) are all highly important factors in climate models [Hansen and Nazarenko 2004] and modelling of global warming effects [Pitari et al. 2015]. Wiscombe (1980) even wrote that "Mie scattering calculations pervade the entire field of atmospheric optics."

While the equations for the Lorenz-Mie coefficients are theoretically unaffected by the absorption properties of the host medium, the distribution of the scattered light is not. This is not so important for polydisperse systems of particles in a weakly absorbing host [Frisvad et al. 2007b; Ma et al. 2019]. However, in cases like random lasers [Luan et al. 2015] and solar cells [Kim et al. 2015], where the host is strongly absorbing and scattering by particles is used to improve efficiency, differences in the distribution of the scattered light could be very important. Imagine for instance that your objective is to direct light into a photovoltaic cell using particle scattering.

The distribution of the scattered light changes when the incident wave becomes inhomogeneous [Frisvad 2018]. Interestingly, a plane wave becomes inhomogeneous upon refraction into an absorbing medium except in the unusual case of incidence along the direction of the surface normal [Fry 1927]. Stratton did not consider an inhomogeneous wave. Early work on scattering by a sphere in an absorbing medium follows Stratton [Galejs 1962; Mundy et al. 1974; Chýlek 1977] and is thus limited to the very rare case of a homogeneous wave in an absorbing medium. More recent work [Belokopytov and Vasil'ev 2006], considers the case of an inhomogeneous wave in a transparent medium. The evanescent wave produced by the phenomenon of total internal reflection is an example of such a case. These waves are particularly important in total internal reflectance microscopy [Prieve and Walz 1993], where the scattering by small particles of an evanescent wave traveling along a flat surface is used to dynamically detect and track the particles. The Lorenz-Mie coefficients $\left(a_{n}\right.$, $b_{n}, c_{n}, d_{n}$ ) are still the same as the ones found by Lorenz (1890), but it turns out that an inhomogeneous wave is particularly effective at exciting the so-called whispering gallery modes leading to microsphere resonators [Belokopytov and Vasil'ev 2006]. In general, the theory of scattering of an inhomogeneous wave by a sphere in an absorbing or a transparent host is not so far from the original Lorenz-Mie theory [Frisvad 2018]. However, the rotational symmetry of the distribution of the scattered light around the direction of the incident light is broken by the wave inhomogeneity.

Microsphere resonators have a surprising number of interesting applications in biosensing, optical signal processing, and development of microlasers [Rakovich and Donegan 2010; Ward and Benson 2011]. The Lorenz-Mie coefficients are directly used to calculate the conditions for an optical resonance [Rakovich and Donegan 2010]. Particles may even be trapped in a resonant cavity. This phenomenon of a radiation pressure laser trap with the ability to levitate a particle is referred to as optical tweezers. In this context, the internal field distribution is again important, and we can calculate this for a particle in a laser beam using generalized LorenzMie theory [Gouesbet 2019]. Optical tweezers have a host of applications and even spurred the rise of single-molecule biophysics [Killian et al. 2018; Polimeno et al. 2018]. The father of optical tweezers, Arthur Ashkin, was awarded the 2018 Nobel prize in physics.

It is interesting to ponder whether Lorenz's unique theoretical analysis of the internal field carries some useful insights just waiting to be collected. In particular, Lorenz's practical approximate formulae for the part of the interior wave due to total internal reflection seem highly interesting [Lorenz 1890, p. 52]. In a geometrical 
optics explanation, these resonances referred to as whispering gallery modes are due to light being trapped inside the particle by total internal reflection. An approximate empirical formula is often used in the analysis of such whispering gallery modes [Rakovich and Donegan 2010]. Lorenz's formulae are self-contained without series expansions, and like the empirical formula they involve an inverse tangent function (in the variables $\delta$ and $\Delta$ ). Thus, they could perhaps lead to a new practical but theoretically founded formula for analyzing the structure of whispering gallery modes.

\section{Lorenz-Mie theory and appearance prediction}

The Lorenz-Mie theory is particularly useful for computing the macroscopic scattering properties of a medium containing a random distribution of scattering particles, a so-called random medium. The seminal textbook in the area of wave propagation in random media is that of Ishimaru (1978). We note that Ishimaru in his latest book describes "the Mie scattering of a dielectric sphere" in more detail and now mentions that "Lorenz gave essentially the same results before Mie's work" [Ishimaru 2017, p. 382]. This means that Lorenz's contribution in all fairness is becoming more widely recognized. The case of dielectric spheres is also precisely the one addressed by Lorenz, while Mie had his focus on conducting spheres. The macroscopic scattering properties would be the phase function $p$ and the scattering coefficient $\mu_{s}$ (which is the same as $h$ for non-absorbing host and particles). The phase function is the far field distribution of the scattered light, while the scattering coefficient is the amount of scattering per unit of distance that light has travelled through the medium. Once such properties are available, we can use them as parameters in the radiative transfer equation [Chandrasekhar 1950; Ishimaru 1978], which together with a theory of light propagation (often geometrical optics and thus ray tracing) forms the basis of material appearance prediction [Frisvad et al. 2007b, 2012].

The link provided by Lorenz-Mie theory between the particle composition of a random medium and its scattering properties and appearance in an image has a broad range of applications. These are not only in atmospheric optics (as mentioned above), but also in biomedical optics [Wang and Wu 2007; Tuchin 2015], hydrological optics [Mobley 1994], heat transfer [Howell et al. 2016], and computer graphics [Callet 1996; Frisvad et al. 2007b]. The Lorenz-Mie theory is prominently featured in seminal textbooks of all these areas except the last. The link from particle composition to appearance becomes important as soon as we are interested in analysis based on imaging modalities. As an example, we can with a noninvasive hyperspectral image of a laser spot reflected from a random medium use Lorenz-Mie theory to estimate size distributions of one or two particle inclusions [Abildgaard et al. 2016]. This inverse use of Lorenz-Mie theory has been around at least since the mid-90s [Box et al. 1992; Mourant et al. 1997], and a recently conducted sensitivity analysis found a good potential for particle sizing in this approach [Postelmans et al. 2018].

Lorenz (1890, p. 56) provides the equations used today for calculating the phase function and the scattering cross section of a spherical particle. The phase function is provided in terms of the transversal components of the wave vector:

$$
\begin{aligned}
& E_{\phi}=-H_{\theta}=\bar{\zeta}_{e}=\frac{\mathrm{i} \sin \phi}{k r} \mathrm{e}^{\mathrm{i}(\omega t-k r)} \sum_{n=1}^{\infty} \frac{2 n+1}{n(n+1)}\left(k_{n} \frac{d P_{n}}{\sin \theta d \theta}+s_{n} \frac{d^{2} P_{n}}{d \theta^{2}}\right) \\
& E_{\theta}=H_{\phi}=\bar{\eta}_{e}=-\frac{\mathrm{i} \cos \phi}{k r} \mathrm{e}^{\mathrm{i}(\omega t-k r)} \sum_{n=1}^{\infty} \frac{2 n+1}{n(n+1)}\left(k_{n} \frac{d^{2} P_{n}}{d \theta^{2}}+s_{n} \frac{d P_{n}}{\sin \theta d \theta}\right) .
\end{aligned}
$$


Lorenz's result is equivalent to the transversal components of the scattered field vectors as found in textbooks deriving the theory based on Maxwell's equations [van de Hulst 1957; Kerker 1969]. The previously mentioned sign difference in Lorenz's expansion coefficients is canceled by Lorenz not using the first order associated Legendre polynomials, since $P_{n}^{1}=-\frac{d P_{n}}{d \theta}$. For the scattering cross section, Lorenz found

$$
C_{s}=\frac{\lambda^{2}}{2 \pi} \sum_{n=1}^{\infty}(2 n+1)\left(\left|k_{n}\right|^{2}+\left|s_{n}\right|^{2}\right)
$$

which is also exactly the same as in modern textbooks given that $k_{n}=-a_{n}$ and $s_{n}=-b_{n}$. Based on Lorenz's exposition, the macroscopic scattering properties of a monodisperse medium with one type of particle of one size are then

$$
\begin{aligned}
p(\theta) & =\frac{\left|S_{1}(\theta)\right|^{2}+\left|S_{2}(\theta)\right|^{2}}{2|k|^{2} C_{s}} \\
S_{1}(\theta) & =\sum_{n=1}^{\infty} \frac{2 n+1}{n(n+1)}\left(k_{n} \frac{d P_{n}}{\sin \theta d \theta}+s_{n} \frac{d^{2} P_{n}}{d \theta^{2}}\right) \\
S_{2}(\theta) & =\sum_{n=1}^{\infty} \frac{2 n+1}{n(n+1)}\left(k_{n} \frac{d^{2} P_{n}}{d \theta^{2}}+s_{n} \frac{d P_{n}}{\sin \theta d \theta}\right) \\
\mu_{s} & =h=N_{p} C_{s} .
\end{aligned}
$$

Remarkably, Lorenz uses these macroscopic scattering properties for precisely the inverse problem discussed above. Toward the end of his memoir, he states the following [Lorenz 1890, pp. 60-61]:

If one has determined wavelengths of a series of absorption lines for a system, these can be ascribed to the reciprocals of the roots in $v_{n}(\beta)=0$, $n=0,1,2, \ldots$, through multiplication by a single constant factor. As this factor is equal to $\frac{N}{2 \pi R}$, it will thus be possible from the refractive index of the system and from its ordinary absorption coefficient to determine all the constants of the system, namely the number of spheres in a unit of volume, the size of the spheres and their refractive index.

Lorenz's suggested approach, which is described in more detail in the concluding pages of the memoir, could easily carry some advantages over the more uninformed data fitting approaches employed in more recent papers.

According to Christiansen (1896) who wrote about Lorenz only a few years after the 1890 treatise, Lorenz had set himself the goal to fully solve the old famous rainbow problem. Thus, Lorenz spent a number of pages on appearance predictions regarding the rainbow (especially pp. 38-42 of the original publication). As an example, Lorenz provides the following formula (Lorenz, 1890, p. 42):

$$
\text { Max. of apparent brightness }=0.06728 \frac{1}{\pi}\left(\frac{R}{\lambda}\right)^{\frac{1}{3}} \text {. }
$$

Suppose we observe a rainbow, then given the relation between wavelengths and perceived colours [Helmholtz 1867], the brightness of the observed colours over the background would reveal the mean radius of the raindrops forming the rainbow. Lorenz cited Helmholtz in his work on dispersion [Lorenz 1883] and was certainly aware of the link between wavelength and perceived colour. The Young-Helmholtz 


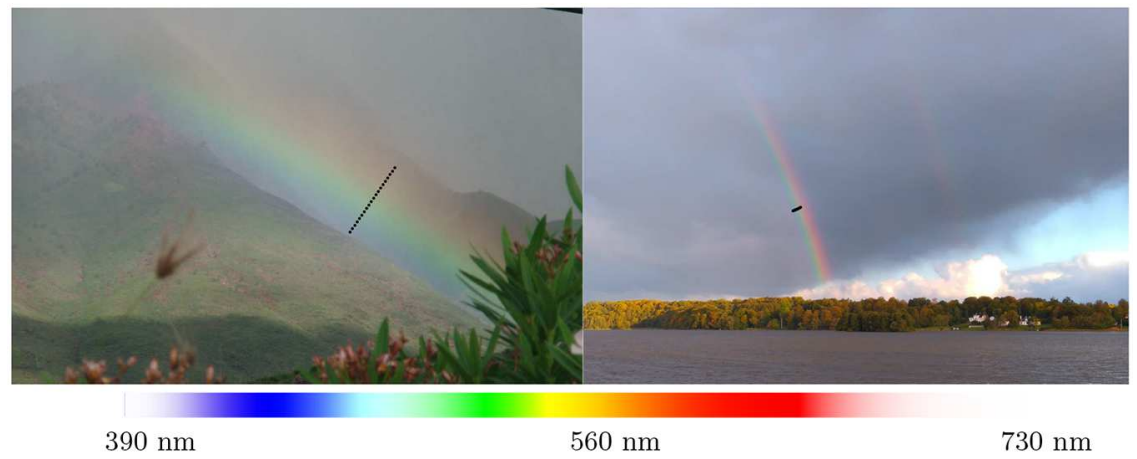

Fig. 3. Two photographs of rainbows and the spectral colours corresponding to visible wavelengths. The background shines through for the wavelengths where the human eye is not very sensitive. We pick pixel samples (dots in images) along a line crossing the rainbow and estimate wavelength and apparent brightness of the scattered light for each pixel by comparing with the spectral colours. Using Lorenz's theory, we find mean waterdrop radius of $\mu=0.26 \mathrm{~mm}(\sigma=0.12 \mathrm{~mm})$ for the left photo and $\mu=0.35 \mathrm{~mm}(\sigma=0.35 \mathrm{~mm})$ for the right photo. These are plausible values corresponding well with the fact that the brightest rainbow pixels in the left image are green while they are red in the right image.

theory of trichromatic colour vision defines three curves that specify the perception of a primary colour as a function of wavelength. The three primary colours are red, green, and blue, and they roughly correspond to the three colour sensitive cones in the human eye. Using psychophysical measurements of these curves [Stiles and Burche 1959; Stockman and Sharpe 2000], we can produce a table of the colours corresponding to different wavelengths. This can then be used together with Lorenz's theory.

Figure 3 is an example of an inverse method where we use Lorenz's rainbow theory for analysis of waterdrop size distributions in rainbows. In addition, Lorenz provided equations for calculating the apparent brightness of the individual spectral colours [Lorenz 1890, Eqs. (a), (b'), and (c')]. This corresponds to appearance prediction. Interestingly, computer graphics today enables us to test the visual quality of historical theories by rendering images based on a given theory [Frisvad et al. 2007a]. We can for example test Aristotle's rainbow theory, which is based on reflecting spherical particles. This can produce a primary rainbow effect at very low computational expense [Frisvad et al. 2007a], but the theory accounts for no specifics in the appearance of rainbows. The visual output of Lorenz's theory has been tested for atmospheric phenomena (sky and rainbow) by Jackèl and Walter (1997) and in detail for rainbows by Sadeghi et al. (2012). In this detailed investigation, the authors found that Lorenz's theory explains most visual rainbow effects. However, for larger waterdrops $(R>0.4 \mathrm{~mm})$, modification is required to account for some visual phenomena as the perfect sphere is no longer as good an approximation.

The ability of the Lorenz-Mie theory to serve as a tool for appearance prediction has surprising quantitative accuracy despite the fact that many particles are not perfect spheres. Surface tension, which forces small liquid particles toward a more spherical shape, is probably one of the reasons behind the success of the theory. When particles are not quite spheres, it mostly affects the accuracy of the directional distribution of the scattered light (the phase function $p$ ). However, in a polydisperse colloid with particles of many different sizes, this is not quite as important as when we consider an engineered material like a solar cell. In polydisperse colloids, an approximate phase function based on the asymmetry parameter $g$ (the mean cosine of the scattering angle) is often sufficient. In case of non-spherical particles, we can 

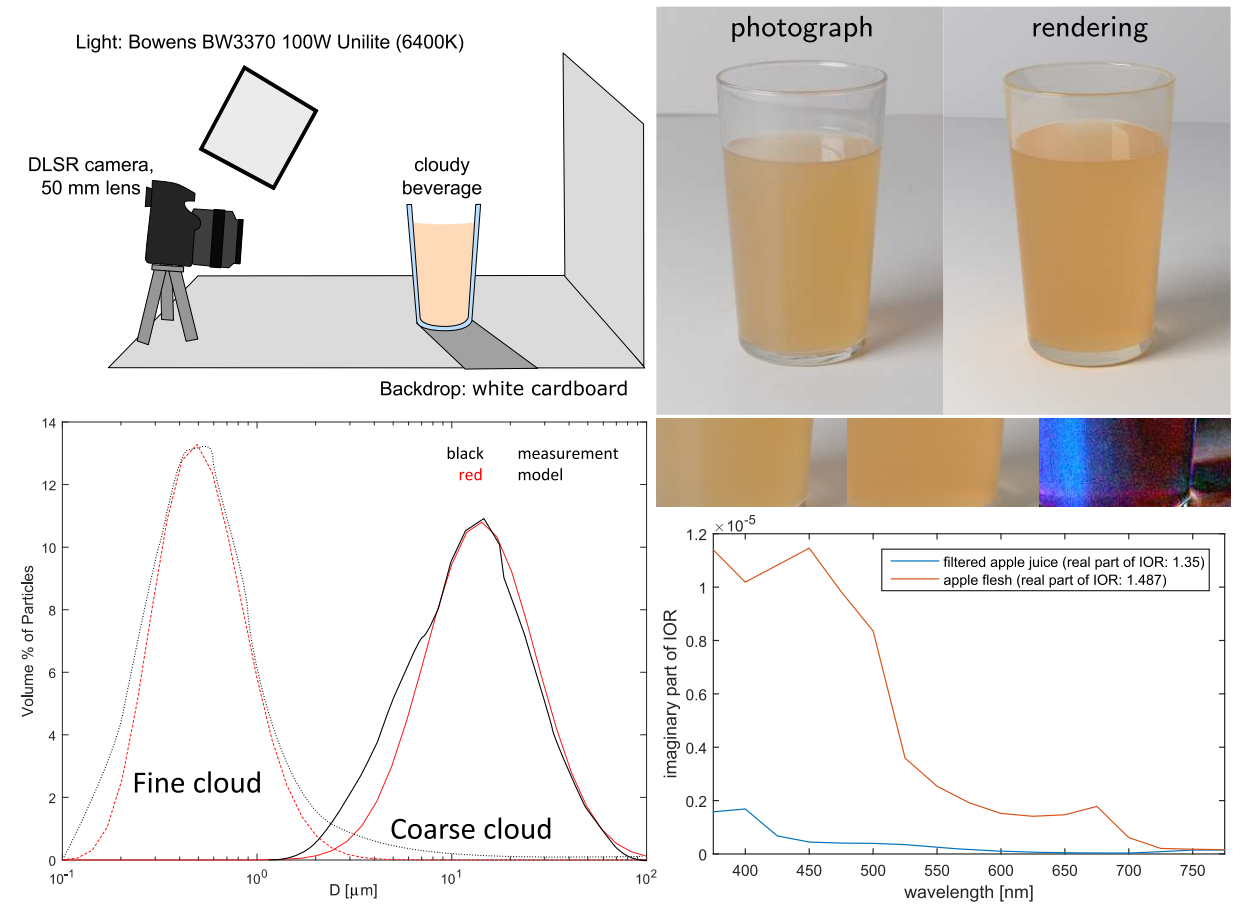

Fig. 4. Let us consider the physical experiment of acquiring a photograph of a polydisperse colloid with a digital camera. The experimental setup is sketched in the upper left corner. We choose cloudy apple juice as our colloid and use an available appearance model based on Lorenz-Mie theory [Dal Corso et al. 2016]. The model produces a bimodal particle size distribution based on the weight-\% of colloidal apple flesh particles (lower left plot, D is particle diameter) and index of refraction (IOR) for the apple juice host and the particles (lower right plot). The appearance predicted by this model is presented in the upper right image. Below photograph and rendering, we have extracted a small part of each image and present a third image: the absolute difference in each colour band multiplied by 10 .

obtain fairly accurate scattering coefficient $\left(\mu_{s}\right)$ and asymmetry parameter $(g)$ using Lorenz-Mie theory on a collection of spheres with volume-to-surface-area ratio that is equivalent to that of the non-spherical particles [Grenfell and Warren 1999]. These two scattering properties, including their wavelength dependence, are critical for the quantitative accuracy of appearance predictions.

Conclusively, we illustrate the quantitative accuracy of the Lorenz-Mie theory when predicting the appearance of a cloudy beverage in Figure 4. As seen in the comparison of the photographed image and the predicted image, appearance differences are mostly a consequence of slight deviations in the glass geometry and reflectance, and in the reflectance of the background made of white cardboard. The colour and translucency of the cloudy beverage are predicted quite convincingly. The advantage of such a model is that we can modify particle size distributions and absorption spectra based on production parameters. In this way, the Lorenz-Mie theory can help predicting the consequences of modified production parameters with respect to product appearance. In addition, we can use calibrated photography (computer vision) to check whether product samples are likely to be according to specification. Lorenz-Mie theory could thus become an important tool in the digitalization of quality assurance and fault detection that we see in industry these years. 


\section{Conclusion}

We firmly believe that the Lorenz-Mie theory will persist for many years to come. In many ways, Lorenz's memoir seems a lost treasure. It is certainly a mathematically dense text, but on the other hand it explains mathematical details that it could take a long time to unearth for the uninitiated. We believe that closer scrutiny of the equations presented by Lorenz could be a fruitful endeavour. In the preceding sections, we have tried to hint at different parts of the text that might be of interest. Lorenz's detailed theoretical investigation of the internal field might be useful with respect to formulation of new practical ways of finding internal resonances in the scattering. The many pages dedicated to approximations could perhaps lead to ingenious inverse methods that we might not otherwise have thought of. In any case, we find it pertinent that Lorenz's 1890 treatise with our translation to English is now more broadly available to the scientific community.

Acknowledgements. The picture in Figure 1 is courtesy of the Royal Library of Copenhagen and included with permission for which we are grateful. The rainbow photographs of Figure 3 were taken by the first author respectively in Maui, Hawaii (left), and Bagsværd, Denmark (right). The reference photograph in Figure 4 also appeared in Dal Corso et al. (2016), but copyright was retained by the authors and not transferred for this publication (and the first author of the present work was one of these authors).

\section{References}

Abildgaard, O. H. A., Frisvad, J. R., Falster, V., Parker, A., Christensen, N. J., Dahl, A. B., and Larsen, R. 2016. Noninvasive particle sizing using camera-based diffuse reflectance spectroscopy. Appl. Opt. 55(14): 3840-3846.

Aden, A. L. and Kerker, M. 1951. Scattering of electromagnetic waves from two concentric spheres. J. Appl. Phys. 22(10): 1242-1246.

Arfken, G. B., Weber, H. J., and Harris, F. E. 2013. Mathemaical Methods for Physicists: A Comprehensive Guide, seventh edn. Academic Print/Elsevier, Massachusetts.

Barton, J. P., Alexander, D. R., and Schaub, S. A. 1988. Internal and near-surface electromagnetic fields for a spherical particle irradiated by a focused laser beam. J. Appl. Phys. 64(4): 1632-1639.

Bateman, H. 1915. The Mathematical Analysis of Electrical and Optical Wave-Motion on the Basis of Maxwell's Equations. Cambridge University Press, Cambridge.

Bauer, G. 1826. Von den Coefficienten der Reihen von Kugelfunctionen einer Variablen. J. Reinen Angew. Math. 56(2): 101-121.

Belokopytov, G. V. and Vasil'ev, E. N. 2006. Scattering of a plane inhomogeneous electromagnetic wave by a spherical particle. Radiophys. Quant. Electr. 49(1): 65-73.

Translated from Izvestiya Vysshikh Uchebnykh Zavedenii, Radiofizika, Vol. 49, No. 1, pp. 72-81, January 2006.

Born, M. and Wolf, E. 1999. Principles of Optics: Electromagnetic Theory of Propagation, Interference and Diffraction of Light, seventh (expanded) edn. Cambridge University Press.

Box, G. P., Sealey, K. M., and Box, M. A. 1992. Inversion of Mie extinction measurements using analytic eigenfunction theory. J. Atmos. Sci. 49(22): 2074-2081.

Callet, P. 1996. Pertinent data for modelling pigmented materials in realistic rendering. Comput. Graph. Forum 15(2): 119-127.

Castanet, G., Delconte, A., Lemoine, F., Mees, L., and Gréhan, G. 2005. Evaluation of temperature gradients within combusting droplets in linear stream using two colors laserinduced fluorescence. Exp. Fluids 39(2): 431-440. 
Chandrasekhar, S. 1950. Radiative Transfer. Oxford University Press, Oxford. Unabridged and slightly revised version published by Dover, New York, 1960.

Chew, H., McNulty, P. J., and Kerker, M. 1976. Model for raman and fluorescent scattering by molecules embedded in small particles. Phys. Rev. A 13(1): 396-404.

Christiansen, C. 1896. Lorenz, ludvig valentin. In Dansk biografisk Lexikon, edited by C. F. Bricka. Copenhagen, 376-381.

Chýlek, P. 1977. Light scattering by small particles in an absorbing medium. J. Opt. Soc. Am. 67(4): 561-563.

Clebsch, A. 1863. Ueber die Reflexion an einer Kugelfläche. J. Reinen Angew. Math. 61: 195-262.

Dal Corso, A., Frisvad, J. R., Kjeldsen, T. K., and Bærentzen, J. A. 2016. Interactive appearance prediction for cloudy beverages. In Workshop on Material Appearance Modeling (MAM 2016). The Eurographics Association, 1-4.

Darrigol, O. 2010. James MacCullagh's ether: An optical route to Maxwell's equations? Eur. Phys. J. H 35(2): 133-172.

Darrigol, O. 2012. A History of Optics from Greek Antiquity to the Nineteenth Century. Oxford University Press, Oxford.

Debye, P. 1909a. Der Lichtdruck auf Kugeln von beliebigem Material. Ann. Phys. 335(11): $57-136$.

Debye, P. 1909b. Näherungsformeln für die Zylinderfunktionen für große Werte des Arguments und unbeschränkt veränderliche Werte des Index. Math. Ann. 67(4): 535-558.

Dusel, P. W., Kerker, M., and Cooke, D. D. 1979. Distribution of absorption centers within irradiated spheres. J. Opt. Soc. Am. 69(1): 55-59.

Frisvad, J. R. 2018. Phase function of a spherical particle when scattering an inhomogeneous electromagnetic plane wave. J. Opt. Soc. Am. A 35(4): 669-680.

Frisvad, J. R., Christensen, N. J., and Falster, P. 2007a. The Aristotelian rainbow: From philosophy to computer graphics. In Proceedings of GRAPHITE 200\%. ACM, $119-128+311$.

Frisvad, J. R., Christensen, N. J., and Jensen, H. W. 2007b. Computing the scattering properties of participating media using Lorenz-Mie theory. ACM Trans. Graph. 26(3): $60: 1-60: 10$.

Frisvad, J. R., Christensen, N. J., and Jensen, H. W. 2012. Predicting the appearance of materials using Lorenz-Mie theory. In The Mie Theory: Basics and Applications, edited by W. Hergert and T. Wriedt. Springer Series in Optical Sciences, Vol. 169. Chap. 4, pp. 101-133.

Fry, T. C. 1927. Plane waves of light I. Electromagnetic behavior. J. Opt. Soc. Am. 15(3): $137-161$.

Galejs, J. 1962. Scattering from a conducting sphere embedded in a semi-infinite dissipative medium. J. Res. Natl. Bur. Stand. D. Radio Propag. 66D(5): 607-612.

Gouesbet, G. 2012. From theories by Lorenz and Mie to ontological underdetermination of theories by experiments. In The Mie Theory: Basics and Applications, edited by W. Hergert and T. Wriedt. Springer Series in Optical Sciences, Vol. 169. Chap. 3, pp. $73-100$.

Gouesbet, G. 2019. Generalized Lorenz-Mie theories and mechanical effects of laser light, on the occasion of Arthur Ashkin's receipt of the 2018 Nobel prize in physics for his pioneering work in optical levitation and manipulation: A review. J. Quant. Spectr. Radiat. Transf. 225: $258-277$.

Gouesbet, G. and Gréhan, G. 1982. Sur la généralisation de la théorie de Lorenz-Mie. J. Opt. 13(2): 97-103.

Gouesbet, G. and Gréhan, G. 2017. Generalized Lorenz-Mie Theories, second edn. Springer, Berlin.

Gouesbet, G., Maheu, B., and Gréhan, G. 1988. Light scattering from a sphere arbitrarily located in a Gaussian beam. J. Opt. Soc. Am. A 5(9): 1427-1443.

Grenfell, T. C. and Warren, S. G. 1999. Representation of a nonspherical ice particle by a collection of independent spheres for scattering and absorption of radiation. J. Geophys. Res. 104(D24): 31,697-31,709. 
Hansen, J. and Nazarenko, L. 2004. Soot climate forcing via snow and ice albedos. Proc. Natl. Acad. Sci. USA 101(2): 423-428.

Helmholtz, H. v. 1867. Handbuch der Physiologischen Optik. Leopold Voss, Leipzig. Three volumes published 1856-1866 and published together in Algemeine Encyklopädie der Physik, Vol. 9, 1867.

Hergert, W. 2012. Gustav Mie: From electromagnetic scattering to an electromagnetic view of matter. In The Mie Theory: Basics and Applications, edited by W. Hergert and T. Wriedt. Springer Series in Optical Sciences, Vol. 169. Chap. 1, pp. 1-51.

Horvath, H. 2009. Gustav Mie and the scattering and absorption of light by particles: Historic developments and basics. J. Quant. Spectr. Radiat. Transf. 110(11): 787-799.

Howell, J. R., Mengüç, M. P., and Siegel, R. 2016. Thermal Radiation Heat Transfer, sixth edn. CRC Press/Taylor \& Francis, Boca Raton.

Ishimaru, A. 1978. Wave Propagation in Random Media. Academic Press, New York. Reissued by IEEE Press and Oxford University Press, 1997.

Ishimaru, A. 2017. Electromagnetic Wave Propagation, Radiation, and Scattering: From Fundamentals to Applications, second edn. John Wiley \& Sons, Hoboken.

Jackèl, D. and Walter, B. 1997. Modeling and rendering of the atmosphere using Miescattering. Comput. Graph. Forum 16(4): 201-210.

Jackson, J. D. and Okun, L. B. 2001. Historical roots of gauge invariance. Rev. Mod. Phys. 73(3): 663-680.

Kaiser, W. 1981. Theorien der Elektrodynamik im 19. Jahrhundert. Gerstenberg, Hildesheim.

Keller, O. 2002. Optical works of L. V. Lorenz. In Progress in Optics, edited by E. Wolf. Vol. 43. Elsevier, Amsterdam, Chap. 3, pp. 195-294.

Kerker, M. 1969. The Scattering of Light and Other Electromagnetic Radiation. Academic Press, New York.

Kerker, M. 1982. Lorenz-Mie scattering by spheres: some newly recognized phenomena. Aeros. Sci. Technol. 1(3): 275-291.

Kerker, M. and Cooke, D. D. 1973. Radiation pressure on absorbing spheres and photophoresis. Appl. Opt. 12(7): 1378-1379.

Kerker, M. and Cooke, D. D. 1982. Photophoretic force on aerosol particles in the freemolecule regime. J. Opt. Soc. Am. 72(9): 1267-1272.

Killian, J. L., Ye, F., and Wang, M. D. 2018. Optical tweezers: A force to be reckoned with. Cell 175(6): 1445-1448.

Kim, I., Lee, K.-S., Lee, T.-S., Jung, D. S., Lee, W.-S., Kim, W. M., and Lee, K.-S. 2015. Size dependence of spherical metal nanoparticles on absorption enhancements of plasmonic organic solar cells. Syn. Metals 199: 174-178.

Kirchhoff, G. 1857. Ueber die Bewegung der Elektricität in Drähten. Ann. Phys. Chem. 176(2): 193-217.

Kragh, H. 2018a. The Lorenz-Lorentz formula: Origin and early history. Substantia 2(2): $7-18$.

Kragh, H. 2018b. Ludvig Lorenz: A Nineteenth-Century Theoretical Physicist. Royal Danish Academy of Sciences and Letters, Copenhagen.

Kragh, H. 2018c. Ludvig Lorenz and his non-Maxwellian electrical theory of light. Phys. Perspect. 20(3): 221-253.

Logan, N. A. 1962. Early history of the Mie solution. J. Opt. Soc. Am. 52(3): 342-343.

Logan, N. A. 1965. Survey of some early studies of the scattering of plane waves by a sphere. Proc. IEEE 53(8): 773-785.

Lorentz, H. A. 1880. Ueber die Beziehung zwischen der Fortpflanzungsgeschwindigkeit des Lichtes und der Körperdichte. Ann. Phys. Chem. 245(4): 641-665.

Lorenz, L. V. 1861. Bestimmung der Schwingungsrichtung des Lichtäthers durch die Reflexion und Brechung des Lichtes. Ann. Phys. Chem. 190(10): 238-250.

Lorenz, L. V. 1863. Ueber die Theorie des Lichts. Ann. Phys. Chem. 194(1): 111-145.

Lorenz, L. V. 1867. On the identity of the vibrations of light with electrical currents. Philos. Mag. 34(230): 287-301.

Lorenz, L. V. 1869. Experimentale og theoretiske Undersøgelser over Legemers Brydningsforhold. Det kongelige danske Videnskabernes Selskabs Skrifter 5(8): 203-248. 
Lorenz, L. V. 1877. Die Lehre vom Licht. Teubner, Leipzig.

Lorenz, L. V. 1880. Ueber die Refractionsconstante. Ann. Phys. Chem. 247(9): 70-103.

Lorenz, L. V. 1883. Theorie der Dispersion. Ann. Phys. Chem. 256(9): 1-21.

Lorenz, L. V. 1890. Lysbevægelser i og uden for en af plane Lysbølger belyst Kugle. Det kongelige danske Videnskabernes Selskabs Skrifter 6(6): 1-62.

Lorenz, L. V. 1898-1904. Oeuvres Scientifiques de L. Lorenz. Vol. 1-2. Lehmann \& Stage, Copenhagen.

Luan, F., Gu, B., Gomes, A. S. L., Yong, K.-T., Wen, S., and Prasad, P. N. 2015. Lasing in nanocomposite random media. Nano Today 10(2): 168-192.

Ma, L. X., Xie, B. W., Wang, C. C., and Liu, L. H. 2019. Radiative transfer in dispersed media: Considering the effect of host medium absorption on particle scattering. J. Quant. Spectr. Radiat. Transf. 230: 24-35.

Maxwell Garnett, J. C. 1904. Colours in metal glasses and in metallic films. Philos. Trans. 203(359-371): 385-420.

McCartney, E. J. 1976. Optics of the Atmosphere: Scattering by Molecules and Particles. John Wiley \& Sons, New York.

Mie, G. 1908. Beiträge zur Optik trüber Medien, speziell kolloidaler Metallösungen. Ann. Phys. 330(3): 377-445.

Mobley, C. D. 1994. Light and Water: Radiative Transfer in Natural Waters. Academic Press, San Diego.

Mourant, J. R., Fuselier, T., Boyer, J., Johnson, T. M., and Bigio, I. J. 1997. Predictions and measurements of scattering and absorption over broad wavelength ranges in tissue phantoms. Appl. Opt. 36(4): 949-957.

Mundy, W. C., Roux, J. A., and Smith, A. M. 1974. Mie scattering by spheres in an absorbing medium. J. Opt. Soc. Am. 64(12): 1593-1597.

Nicholson, J. W. 1910. The asymptotic expansions of Bessel functions. Philos. Mag. 19(110): 228-249.

Pitari, G., Di Genova, G., and De Luca, N. 2015. A modelling study of the impact of onroad diesel emissions on arctic black carbon and solar radiation transfer. Atmosphere 6(3): 318-340.

Polimeno, P., Magazzù, A., Iatì, M. A., Patti, F., Saija, R., Boschi, C. D. E., Donato, M. G., Gucciardi, P. G., Jones, P. H., Volpe, G., and Maragò, O. M. 2018. Optical tweezers and their applications. J. Quant. Spectr. Radiat. Transf. 218: 131-150.

Postelmans, A., Aernouts, B., and Saeys, W. 2018. Estimation of particle size distributions from bulk scattering spectra: sensitivity to distribution type and spectral noise. Opt. Express 26(12): 15015-15038.

Poynting, J. H. 1884. On the transfer of energy in the electromagnetic field. Philos. Trans. 175: $343-361$.

Prieve, D. C. and Walz, J. Y. 1993. Scattering of an evanescent surface wave by a microscopic dielectric sphere. Appl. Opt. 32(9): 1629-1641.

Rakovich, Y. P. and Donegan, J. F. 2010. Photonic atoms and molecules. Laser Photonics Rev. 4(2): 179-191.

Rayleigh, Lord. 1871a. On the light from the sky, its polarization and colours. Philos. Mag. 41(271): 107-120.

Rayleigh, Lord. 1871b. On the light from the sky, its polarization and colours. Philos. Mag. 41(273): 274-279.

Rayleigh, Lord. 1871c. On the scattering of light by small particles. Philos. Mag. 41(275): 447-454.

Rayleigh, Lord. 1881. On the electromagnetic theory of light. Philos. Mag. 12(73): 81-101.

Rayleigh, Lord. 1899. On the transmission of light through an atmosphere containing small particles in suspension, and on the origin of the blue of the sky. Philos. Mag. 47(287): 375-384.

Sadeghi, I., Muñoz, A., Laven, P., Jarosz, W., Seron, F., Gutierrez, D., and Jensen, H. W. 2012. Physically-based simulation of rainbows. ACM Trans. Graph. 31(1): 3:1-3:12.

Schaub, S. A., Alexander, D. R., Barton, J. P., and Emanuel, M. A. 1989. Focused laser beam interactions with methanol droplets: effects of relative beam diameter. Appl. Opt. 28(9): 1666-1669. 
Schuster, A. 1909. An Introduction to the Theory of Optics. Edward Arnold, London.

Stiles, W. S. and Burche, J. M. 1959. N.P.L. colour-matching investigation: Final report (1958). Opt. Acta 6: 1-26.

Stockman, A. and Sharpe, L. T. 2000. The spectral sensitivities of the middle- and longwavelength-sensitive cones derived from measurements in observers of known genotype. Vis. Res. 40(13): 1711-1737.

Stratton, J. A. 1941. Electromagn. Theory. McGraw-Hill, New York.

Todhunter, I. 1893. A History of the Theory of Elasticity and of the Strength of Materials: From Galilei to the Present Time. Vol. II. Saint-Venant to Lord Kelvin. Cambridge University Press, Cambridge.

Tuchin, V. 2015. Tissue Optics: Light Scattering Models and Instruments for Medical Diagnosis, third edn. SPIE Press, Washington.

van de Hulst, H. C. 1957. Light Scattering by Small Particles. John Wiley \& Sons, New York.

Vizgin, V. P. 1994. Unified Field Theories: in the First Third of the 20th Century. Birkhäuser, Basel.

Wait, J. R. 1962. Electromagnetic scattering from a radially inhomogeneous sphere. Appl. Sci. Res., Section B 10(5-6): 441-450.

Wait, J. R. 1998. The ancient and modern history of EM ground-wave propagation. IEEE Antennas Propag. Mag. 40(5): 7-24.

Wang, L. V. and Wu, H. 2007. Biomedical Optics: Principles and Imaging. John Wiley \& Sons, Hoboken.

Ward, J. and Benson, O. 2011. Wgm microresonators: sensing, lasing and fundamental optics with microspheres. Laser Photon. Rev. 5(4): 553-570.

Watson, G. N. 1922. A Treatise on the Theory of Bessel Functions. Cambridge University Press, Cambridge.

Whittaker, E. 1958. A History of the Theories of Aether and Electricity: Vol. I: The Classical Theories, 2nd revised edn. Thomas Nelson, London.

Wiscombe, W. J. 1980. Improved Mie scattering algorithms. Appl. Opt. 19(9): 1505-1509.

Wriedt, T. 2012. Mie theory: A review. In The Mie Theory: Basics and Applications, edited by W. Hergert and T. Wriedt. Springer Series in Optical Sciences, Vol. 169. Chap. 2, pp. $53-71$. 